\title{
Crucial role of androgen receptor in resistance and endurance trainings-induced muscle hypertrophy through IGF-1/IGF-1R- PI3K/Akt- mTOR pathway
}

\author{
Lijun YIN, Lin LU, Xiaojing LIN and Xiaohui WANG*
}

\begin{abstract}
Background: Androgen receptor (AR) has been reported to play vital roles in exercise-induced increase of muscle mass in rats, but needs to be further verified and the mechanism behind remains unclear. As AR target genes, insulin growth factor-1 (IGF-1) and IGF-1 receptor (IGF-1R) promote muscle hypertrophy through activating PI3K Akt- mammalian target of rapamycin (mTOR) pathway, a classic pathway of muscle hypertrophy. So the main purpose of this study was using AR antagonist flutamide to demonstrate AR's effect on training-induced muscle hypertrophy and its possible mechanism: IGF-1/IGF-1R- PI3K/Akt- mTOR pathway?

Methods: Forty-eight Sprague Dawley male rats aged 7 weeks were randomly divided into six groups: control (C), flutamide $(F)$, resistance training $(R)$, resistance training plus flutamide $(R+F)$, endurance training $(E)$, and endurance training plus flutamide $(E+F)$ groups. Flutamide was used to block $A R$ in rats. Rats in $R$ and $R+F$ groups fulfilled 3 weeks of ladder climbing with progressively increased load, while $E$ and $E+F$ rats completed 3-week moderate intensity aerobic exercise on a treadmill. The relative muscle mass (muscle mass/body weight) of rats was detected. Serum levels of testosterone and IGF-1 of rats were determined by ELISA, and mRNA levels of IGF-1R and mTOR in muscles by real-time PCR. Protein levels of AR, IGF-1, IGF-1R, mTOR, PI3K, Akt, p-PI3K and p-Akt in muscles were detected by Western blot.

Results: (1) The training-induced rise in the relative muscle mass and the expression levels of AR were only found in the gastrocnemius of $R$ rats and in the soleus of $E$ rats (selective muscle hypertrophy), which were blocked by flutamide. (2) Serum testosterone in the $R$ and $E$ rat were increased, and flutamide exerted no effect. (3) The levels of IGF-1, IGF-1R and mTOR as well as the activities of PI3K and Akt were enhanced selectively (in the gastrocnemius of R rats and in the soleus of $E$ rats), which were reduced by flutamide. Conclusions: AR exerted an essential role in both resistance training and endurance training-induced muscle hypertrophy, which was mediated at least partly through IGF-1/IGF-1R- PI3K/AktmTOR pathway.
\end{abstract}

Keywords: Androgen receptor, Muscle hypertrophy, Training, IGF-1, IGF-1R, PI3K/Akt/mTOR

\section{Introduction}

Testosterone is widely reported to affect muscle mass and exercise capacity, with high level testosterone promoting,

\footnotetext{
* Correspondence: wangpan96@126.com

Department of Kinesiology, Shanghai University of Sport, 188 Hengren Road, Yangpu District, Shanghai 200438, People's Republic of China
}

while low level declining muscle mass and exercise capacity. Exogenous androgen administration significantly facilitated the protein synthesis, prevented muscle proteolysis and increased the muscle mass and strength via the mediation of androgen receptor (AR) [1]. Due to the severe side-effects (cardiovascular events and benign

(c) The Author(s). 2020 Open Access This article is licensed under a Creative Commons Attribution 4.0 International License, which permits use, sharing, adaptation, distribution and reproduction in any medium or format, as long as you give appropriate credit to the original author(s) and the source, provide a link to the Creative Commons licence, and indicate if changes were made. The images or other third party material in this article are included in the article's Creative Commons licence, unless indicated otherwise in a credit line to the material. If material is not included in the article's Creative Commons licence and your intended use is not permitted by statutory regulation or exceeds the permitted use, you will need to obtain permission directly from the copyright holder. To view a copy of this licence, visit http://creativecommons.org/licenses/by/4.0/ The Creative Commons Public Domain Dedication waiver (http://creativecommons.org/publicdomain/zero/1.0/) applies to the data made available in this article, unless otherwise stated in a credit line to the data. 
prostatic hyperplasia) resulted from long-term or large dose of androgen administration, androgen supplementation is strictly limited [2], and selective androgen receptor modulators (SARMs) especially the non-steroidal tissuespecific SARMs were used currently to activate AR in specific tissue. Sufficient evidences demonstrated the enhancement of lean mass and strength obtained by SARMs in dystrophy muscle of female elderly with sarcopenia [3] and mice with myotonic dystrophy [4]. On the contrary, global AR gene knockout (ARKO) and specific skeletal muscle tissue ARKO mice showed alterations in the muscle mass (such as gastrocnemius, quadriceps and soleus) $[5,6]$, muscle glycogen [7] and exercise performance. The important role of AR in the increases of muscle mass and exercise performance at untrained state was demonstrated [5, 8-11], but whether AR exerted crucial role in exercise-induced muscle hypertrophy in vivo have not been thoroughly verified. To our best knowledge, until now no ARKO model mice have been used to explore AR's role in exercise-induced changes in muscle mass and performance, moreover, ARKO mice could not be obtained commercially. Thus, AR specific antagonist flutamide is still widely employed to clarify AR's role $[12,13]$.

The molecular mechanisms by which AR regulates exercise-induced alterations in muscle mass remain largely unknown. It is widely accepted that insulin-like growth factor 1 (IGF-1) plays vital roles in the proliferation of myoblasts and muscle hypertrophy. Previous studies have observed the significant increase of muscle mass in mice with overexpression of IGF-1 and also revealed the decrease of IGF-1 in mice with attenuated muscle mass caused by malnutrition, diseases or aging. The biological functions of IGF-1 are mediated by IGF-1R. Moreover, AR's effects on facilitating untrained muscle hypertrophy are at least partly associated with IGF-1/IGF-1R, reflected as androgen supplementation greatly increased the muscle size accompanied with an increase of IGF-1 expression [14], while AR blockage significantly blunted the testosterone-induced myotube formation and enlarged diameters, and reduced the mRNA level of IGF-1R in C2C12 myoblast [15].

IGF-1/IGF-1R contributes to muscle hypertrophy on untrained state mainly through activating phosphatidylinositol 3 kinase (PI3K)/protein kinase B (Akt)- mammalian target of rapamycin (mTOR) pathway [16-18], evidenced as IGF1 induced-growth promotion of skeletal muscle could be greatly inhibited by PI3K inhibitor LY294002 or Akt inhibitor KP372-1 [19]. PI3K/Akt- mTOR pathway is activated by phosphorylating PI3K, Akt and mTOR successively after PI3K translocation to phosphorylated insulin receptor substrate 1 [18], then exerted its vital roles in regulating muscle development and hypertrophy by promoting proliferation and muscle protein synthesis and preventing muscle degradation as well [20]. Tight relationship of AR with PI3K/ Akt- mTOR pathway has been revealed by considerable amount of evidences, for example, PI3K/Akt inhibitor significantly reduced testosterone-induced muscle hypertrophy, and in turn, AR antagonist flutamide offset the increase of muscle mass caused by androgen supplementation accompanied with the decreased levels of IGF-1R and p-Akt [21, 22], which indicated that androgen/AR-induced muscle hypertrophy in untrained participants was at least partly associated with the activation of IGF-1- Akt/mTOR pathway. But whether the effects of AR on muscle hypertrophy at trained state was mediated by IGF-1- Akt/mTOR pathway remains unproved.

Our previous studies using mechanical stretch of myoblasts to mimic muscle movement have revealed an indispensable role of AR and its mechanism in vitro -- through the mediation of IGF-1/IGF-1R- PI3K-mTOR pathway, according to two facts: (1) mechanical stretch-induced pro-proliferation of $\mathrm{C} 2 \mathrm{C} 12$ myoblasts (highly expressed AR) was reversed by flutamide, or IGF-1R blockade or PI3K/Akt inhibition [23]; (2) stretch-induced proproliferation was greatly attenuated in L6 myoblasts (without $\mathrm{AR}$ expression) compared to $\mathrm{C} 2 \mathrm{C} 12$ myoblasts, and the proliferation of $\mathrm{L} 6$ myoblast was promoted by either AR over-expression or IGF-1 supplement, then inducing the activation of IGF-1R- PI3K- mTOR pathway [24].

Therefore, in the current study, flutamide was used to block AR signaling in male rats to confirm the positive effect of AR on exercise-induced muscle hypertrophy in vivo and further explore its mechanisms: IGF-1/IGF1R- PI3K/Akt- mTOR pathway?

\section{Materials and methods}

\section{Animals and grouping}

Forty-eight Sprague-Dawley (SD) male rats aged 7 weeks (170-190 g body weight) were purchased from Beijing Vital River laboratory Animal Technology Co. Ltd., and housed under standard specific pathogen free (SPF) conditions in a temperature and humility-controlled room on a 12-h light:12-h dark cycle. Food and water were freely available throughout the study. The animal protocol was approved and all the experimental procedures were supervised by the Ethics Committee of Shanghai University of Sport (No. 2018002). After acclimating to laboratory conditions for 3 days, rats were randomly and evenly divided into six groups: control (C), AR antagonist flutamide embedded $(\mathrm{F})$, resistance training $(\mathrm{R})$, resistance training plus flutamide $(R+F)$, endurance exercise $(E)$ and endurance exercise plus flutamide $(\mathrm{E}+\mathrm{F})$.

\section{Flutamide treatment}

Rats in $F$ group, $E+F$ group and $R+F$ group were anesthetized and embedded with flutamide releasing pellet $(50 \mathrm{mg} /$ pellet, evenly releases for 21 days, Innovative Research of America, USA.) prior to exercise intervention. Briefly, after anesthesia, an almost 0.5 
cm long incision was cut on the neck, and pellet was implanted into the incision site, then gently sutured the incision. Rats in $\mathrm{C}$ group also underwent the surgery but no pellet embedded.

\section{Exercise protocol}

As shown in Fig. 1, after one-day adaptation to endurance or resistance training, rats in $\mathrm{E}$ and $\mathrm{E}+\mathrm{F}$ groups participated in a moderate intensity aerobic exercise on a treadmill at a speed of $20 \mathrm{~m} / \mathrm{min}$ for 1 hour each time, while the rats in $\mathrm{R}$ and $\mathrm{R}+\mathrm{F}$ groups underwent resistance training, which comprises three sets of ladder climbing with progressively incremental load. Briefly, rats start climbing at the bottom of the ladder $(1 \mathrm{~m}$ in height, $2 \mathrm{~cm}$ distance from each stairs and a slope of $85^{\circ}$ ) with progressively increased load on tail (the initial weight attached to tail was $40 \%$ of body weight, and increased by $10 \%$ every 2 days until reached $120 \%$ of body weight). Every set consists of four repetitions and three sets $(20 \mathrm{~s}$ interval period between sets) in each time. Resistance or endurance training lasts for 3 weeks and 6 days per week.

\section{Detection of serum testosterone and IGF-1 by ELISA}

Serum testosterone and IGF-1 levels of rats were measured by ELISA according to manufacturer's instructions. The intra- and inter-assay coefficients of variation (CV) were less than 2.9 and $6.8 \%$ for detecting testosterone (KGE010, R\&D systems, Minnesota Minneapolis, USA) as well as 4.1 and $4.3 \%$ for IGF-1 kit (MG100, R\&D systems, Minnesota Minneapolis, USA).

\section{Quantitative real-time PCR}

Rats were anaesthetized at $36 \mathrm{~h}$ after the last exercise, gastrocnemius and soleus were collected and frozen in liquid nitrogen then stored at $-80^{\circ} \mathrm{C}$ until analyzed or immediately processed as the following process. Total RNA was extracted using Trizol reagent (Invitrogen, CA,
USA), the first strand cDNA was synthesized by Revert Aid First Stand cDNA Synthesis Kit (Thermo Scientific, MA, USA) in accordance with the manufacturer's instructions. Primers for amplification genes of IGF-1R (sense: 5'-AAA CGC TGA CCT CTG TTA CCT-3', antisense: 5'-ACG CCT TTG TAG TAG TAG TGT CG-3'), mTOR (sense: 5'-GAT ACG CCG TCA TTC CTC-3', antisense: $5^{\prime}$-TGC TCA AAC ACC TCC ACC$3^{\prime}$ ) and GAPDH (sense: $5^{\prime}$-GCT GAG TAT GTC GTG GAG-3', antisense: $5^{\prime}$-TCT TCT GAG TGG CAG TGA T-3') were synthesized by Sangon Biotech Co, Ltd. Shanghai. $50 \mathrm{ng}$ of cDNA templates were added into FastStart universal SYBR Green Master (Roche company, Switzerland) to amplify the genes above, and the amplification conditions for these genes were the same: $10 \mathrm{~min}$ denaturation at $95^{\circ} \mathrm{C}$ followed by $40 \mathrm{cy}$ cles of $15 \mathrm{~s}$ denaturation at $95^{\circ} \mathrm{C}, 60 \mathrm{~s}$ annealing and elongation at $60^{\circ} \mathrm{C}$. The mRNA values of IGF-1R and mTOR of samples were corrected by that of the internal control of GAPDH and shown as the ratios of target genes to GAPDH.

\section{Western blot}

About $50 \mathrm{mg}$ of gastrocnemius and soleus were cut into pieces and homogenized after adding $500 \mu \mathrm{L}$ of radioimmunoprecipitation assay (RIPA) buffer containing phenylmethanesulfonyl fluoride (PMSF) (Beyotime Biotechnology, China) (RIPA:PMSF $=100: 1$ ) to extract total protein. After sonication on the ice, lysates were centrifuged at $14000 \mathrm{rpm}$ for $20 \mathrm{~min}$ at $4{ }^{\circ} \mathrm{C}$. Then supernatants were collected and protein concentration was determined by BCA protein assay kit (Beyotime Biotechnology, Shanghai, China). Extracts $(50 \mu \mathrm{g})$ of gastrocnemius or soleus was fractionated on $10 \%$ SDS-PAGE gels, then electro-transferred onto nitrocellulose membranes. After blocking with $5 \%$ nonfat milk for $2 \mathrm{~h}$, the nitrocellulose membranes

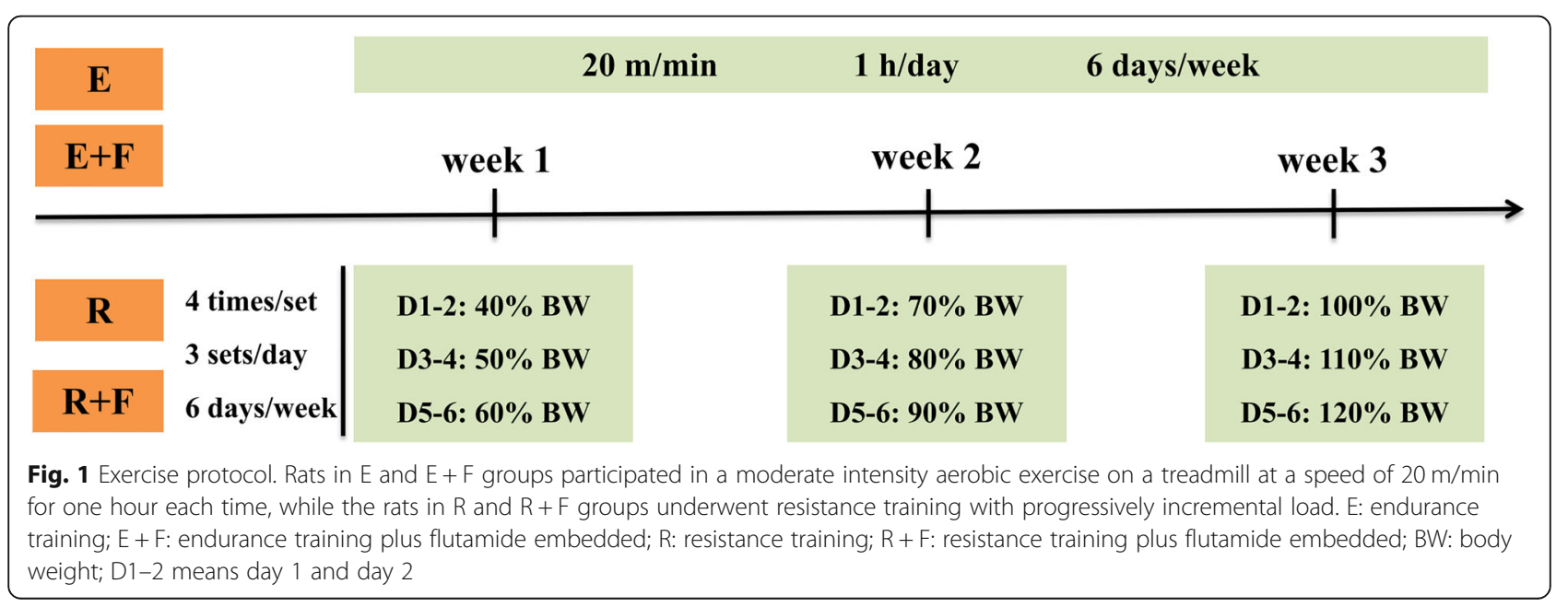



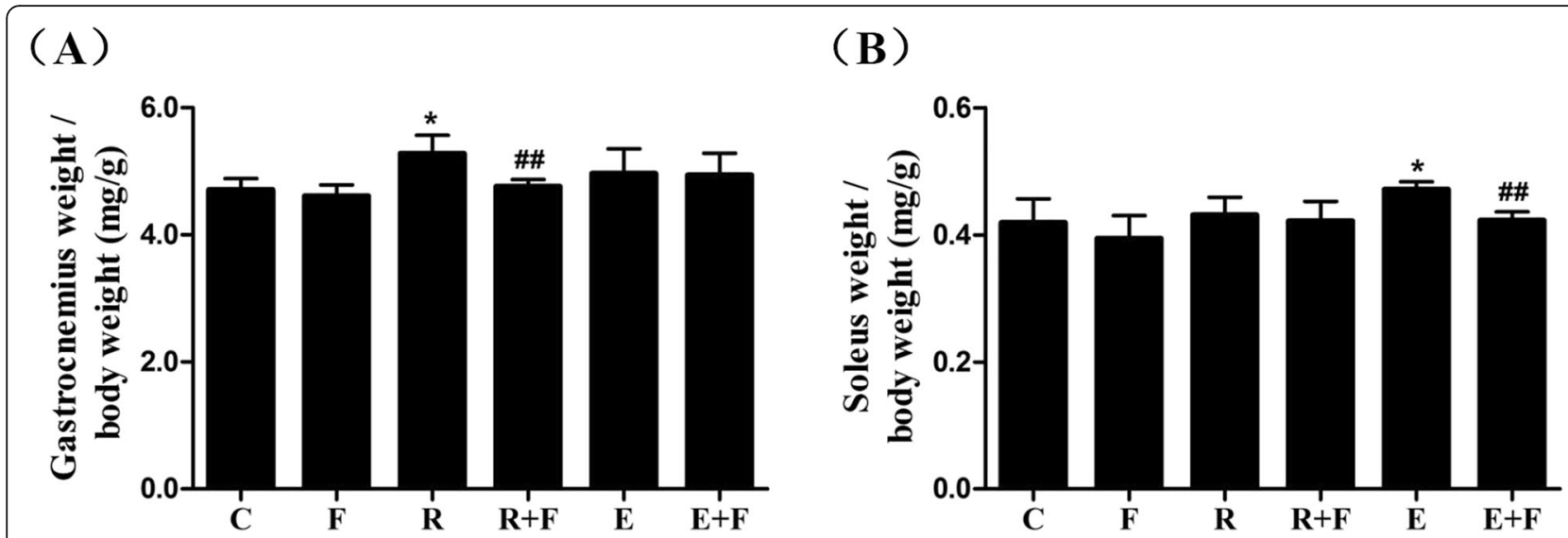

Fig. 2 Flutamide reversed the training-induced selective muscle hypertrophy. Resistance training and endurance training increased the relative muscle mass of gastrocnemius (a) and soleus (b) respectively, which was reversed by flutamide treatment. The ratio of muscle weight with body weight is considered as the relative muscle mass. C: control; F: flutamide embedded; R: resistance training; $R+F$ : resistance plus flutamide embedded; E: endurance training; E + F: endurance plus flutamide embedded. ${ }^{*} P<0.05$ vs $C_{;}{ }^{\# \#} P<0.01$ vs the relative training group

were incubated overnight at $4{ }^{\circ} \mathrm{C}$ with primary antibodies against AR and IGF-1R (both 1:500, Santa Cruz Biotechnology, CA, USA), PI3K, Akt, mTOR, pPI3K and p-Akt (all 1:1000, Cell Signaling Technology, MA, USA). After washing three times with Trisbuffered saline with $0.1 \%$ Tween 20 (TBST), each time for $5 \mathrm{~min}$, the bands were incubated with horseradish peroxidase (HRP) conjugated secondary antibodies (Santa Cruz Biotechnology, CA, USA) for $1 \mathrm{~h}$ at room temperature. Then, the bands were washed again as described above, developed with Immbilon Western chemiluminescent HRP substrate (Millipore, MA, USA), and visualized by automatic chemiluminescence image analysis system (Tanon Biotechnology, Shanghai, China). The density of blot was determined using Bio-image software (Tanon Biotechnology, Shanghai, China) and normalized against GAPDH.

\section{Statistical analysis}

Statistical analysis of data was performed using SPSS for Windows 19.0 software package (IBM Corporation, Armonk, NY, USA). All data were presented as Mean \pm SD. Statistical difference for $C, F, R$ and $\mathrm{R}+\mathrm{F}$ groups, as well as the $\mathrm{C}, \mathrm{F}, \mathrm{E}$ and $\mathrm{E}+\mathrm{F}$ groups were determined by one-way analysis of variance (ANOVA) and post hoc comparison using least significant difference (LSD)- $t$ test. The level of statistical significance was set as $p<0.05$.

\section{Results}

Flutamide reversed the training-induced selective muscle hypertrophy

Compared with control, the relative muscle mass of gastrocnemius but not soleus was increased in $R$ rats (Fig. 2a), while enhanced muscle mass was observed only in the soleus of E rats (Fig. 2b), indicating a selective muscle hypertrophy induced by trainings (means resistance training mainly results in hypertrophy of fast switch muscle such as gastrocnemius, while endurance training mainly induces hypertrophy of slow switch muscle such as soleus). Furthermore, flutamide could reverse the training-induced selective muscle hypertrophy (Fig. 2).

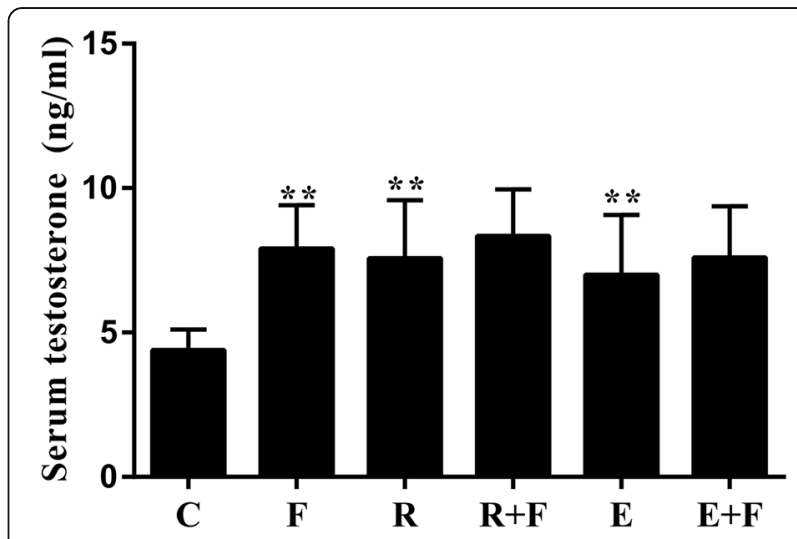

Fig. $\mathbf{3}$ Effects of trainings and flutamide on the serum testosterone. Both resistance training and endurance training increased the serum testosterone concentration. Flutamide did not alter the level of serum testosterone in either resistance training or endurance training rats although an increase of serum testosterone was found in sedentary rats. The levels of serum testosterone in rats were detected by ELISA. C: control; F: flutamide embedded; R: resistance training; $R+F$ : resistance training plus flutamide embedded; $E$ : endurance training; $E+F$ : endurance training plus flutamide embedded. ${ }^{* *} P<0.01$ vs $C$ 


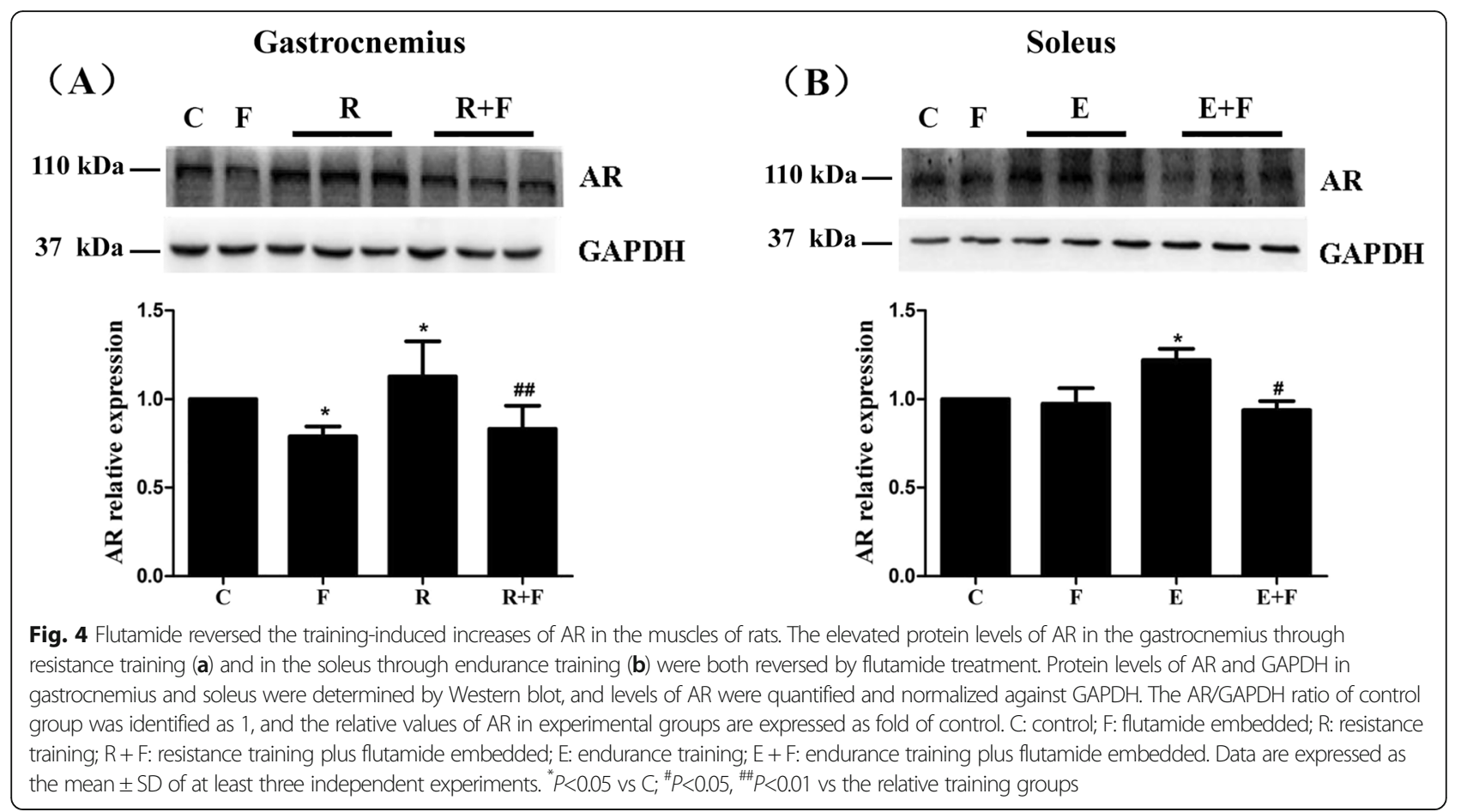

Flutamide reversed the training-induced selective increase of AR protein level in muscles instead of promotion of testosterone in serum

The serum levels of testosterone in $\mathrm{R}$ and $\mathrm{E}$ rats were significantly increased compared to control, and blocked AR by flutamide did not change the levels of serum testosterone in the training rats, while flutamide elevated serum testosterone of the untrained control rats (Fig. 3).

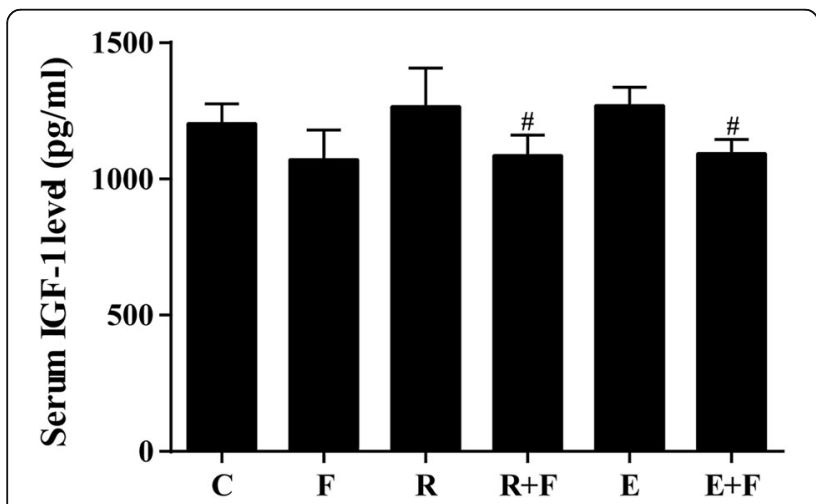

Fig. $\mathbf{5}$ Flutamide lowered the serum IGF-1 concentration of training rats. Flutamide decreased the serum concentration of IGF-1 in both resistance training and endurance training rats although no change was found in rats with flutamide treatment or training alone. C: control; F: flutamide embedded; R: resistance training; R + F: resistance training plus flutamide embedded; E: endurance training; $\mathrm{E}+\mathrm{F}$ : endurance training plus flutamide embedded. ${ }^{\#} P<0.05$ vs the relative training groups
Accompanied with the training-induced selective muscle hypertrophy, AR also showed a selective increase, characteristic of up-regulated AR only in gastrocnemius of $R$ rats (Fig. 4a) and in soleus of $E$ rats (Fig. 4b) compared with control rats, while no alteration of AR was observed in non-hypertrophic muscles including the soleus of $\mathrm{R}$ rats and the gastrocnemius of $\mathrm{E}$ rats (data not shown). Furthermore, the selective increases of AR in the hypertrophy muscles of $\mathrm{R}$ and $\mathrm{E}$ rats were noticeably blocked by flutamide, and an attenuation of AR in gastrocnemius but not in soleus of $\mathrm{F}$ rats was found compared with control (Fig. 4).

Table 1 Flutamide reversed the enhancement of IGF-1R and mTOR at mRNA levels induced by trainings

\begin{tabular}{lllllll}
\hline & $n$ & \multicolumn{2}{l}{ Gastrocnemius } & & \multicolumn{2}{l}{ Soleus } \\
& & IGF-1R & mTOR & & IGF-1R & mTOR \\
\hline C & 7 & $1.00 \pm 0.23$ & $1.00 \pm 0.18$ & & $1.00 \pm 0.20$ & $1.00 \pm 0.21$ \\
F & 6 & $0.88 \pm 0.11$ & $0.91 \pm 0.11$ & & $0.81 \pm 0.14$ & $0.85 \pm 0.14$ \\
R & 8 & $1.32 \pm 0.21^{*}$ & $1.43 \pm 0.10^{* *}$ & $/$ & $/$ \\
R+F & 8 & $0.96 \pm 0.06^{\# \#}$ & $0.97 \pm 0.16^{\# \#}$ & $/$ & $/$ \\
E & 8 & $/$ & $/$ & & $1.30 \pm 0.20^{*}$ & $1.32 \pm 0.28^{*}$ \\
E+F & 7 & $/$ & $/$ & $0.98 \pm 0.18^{\#}$ & $0.94 \pm 0.27^{\# \#}$
\end{tabular}

Note: The increased mRNA levels of IGF-1R and mTOR were found in the gastrocnemius of resistance training rats and in the soleus of endurance training rats. Flutamide reversed the trainings-induced changes in IGF-1R and mTOR at mRNA level. C Control, $F$ Flutamide embedded, $R$ Resistance training, $R+F$ Resistance training plus flutamide embedded, $E$ Endurance training, $E+F$ Endurance training plus flutamide embedded. ${ }^{*} P<0.05,{ }^{* *} P<0.01$ vs $C$; ${ }^{\#} P<0.05$, ${ }^{\# \#} P<0.01$ vs the relative training groups 


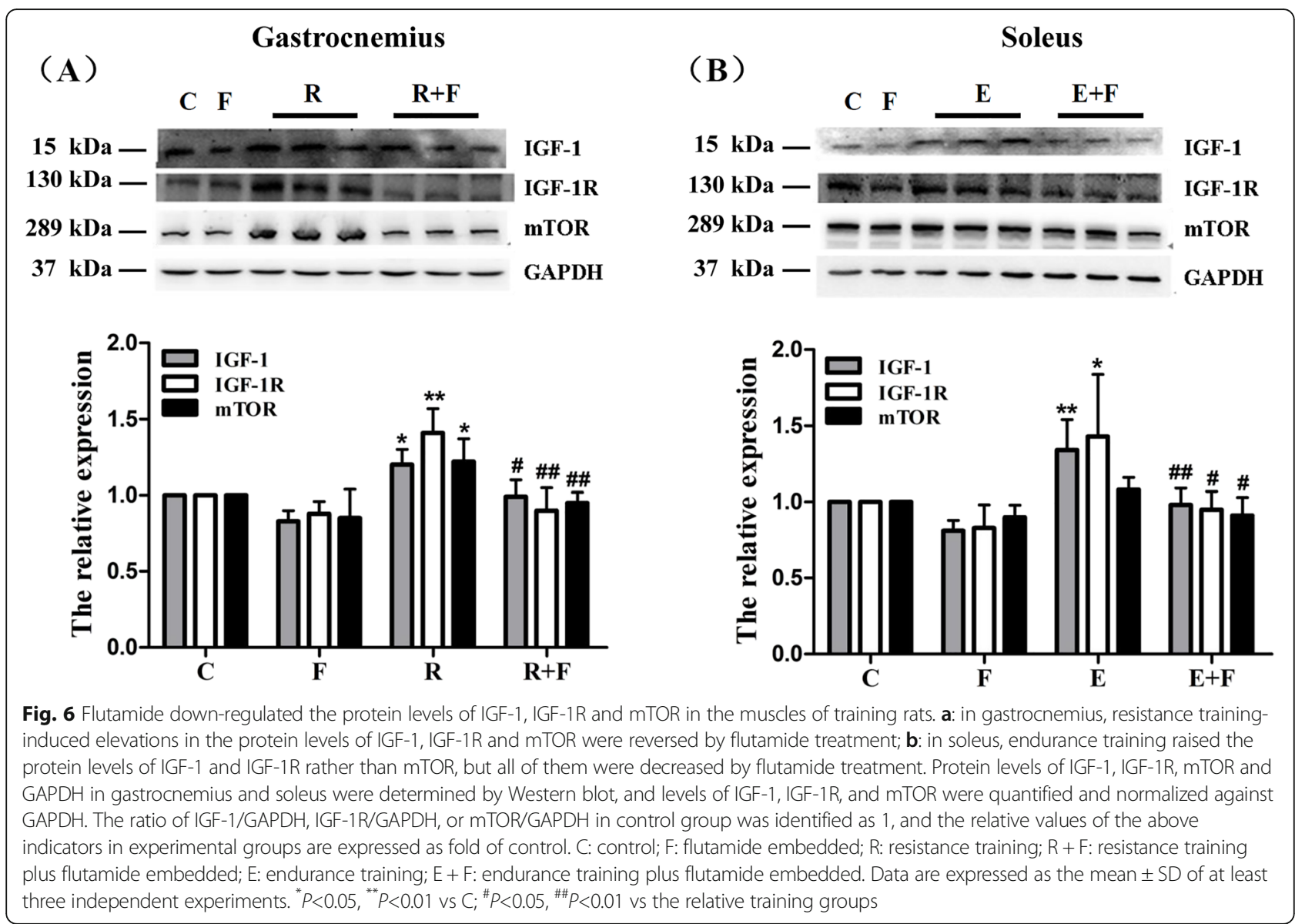

Flutamide lowered serum IGF-1 of the trained rats

As shown in Fig. 5, obvious attenuation of serum IGF-1 concentration was found in $R+F$ rats compared with $R$ rats and in $E+F$ rats compared with $E$ rats, although no noticeable alteration of IGF-1 was induced by resistance or endurance training.

Flutamide reversed the training-induced selective enhancement of IGF-1R and mTOR

As shown in Table 1, significant increases of the mRNA levels of IGF-1R and mTOR were observed in gastrocnemius of $R$ rats or in soleus of $E$ rats compared with control rats, and moreover, the two trainings-induced increases in IGF-1R and mTOR were reversed by flutamide.

Similar results were obtained on the protein levels of IGF-1, IGF-1R and mTOR. Compared with control rats, the protein levels of IGF-1, IGF-1R and mTOR were enhanced only in gastrocnemius of $R$ rats or soleus of $\mathrm{E}$ rats (but no change of mTOR in E rats) (Fig. 6), and no alterations of IGF-1, IGF-1R and mTOR were found in non-hypertrophic muscles including the soleus of $\mathrm{R}$ rats and the gastrocnemius of $E$ rats (data not shown), indicated selective increases of IGF-1, IGF-1R and mTOR at protein levels in the hypertrophy muscle by the two trainings. Furthermore, the protein levels of IGF-1, IGF$1 \mathrm{R}$ and mTOR were down-regulated in $\mathrm{R}+\mathrm{F}$ rats compared with $\mathrm{R}$ rats and in $\mathrm{E}+\mathrm{F}$ rats compared with $\mathrm{E}$ rats (Fig. 6).

Flutamide reversed the training-induced selective increases in expressions and activities of PI3K and Akt Significant increases in activities rather than expression levels of PI3K and Akt were observed in the gastrocnemius of $\mathrm{R}$ rats (Fig. 7a) or in the soleus of $\mathrm{E}$ rats (Fig. $7 \mathrm{~b}$ ) compared with control rats, and no changes were observed in the activities and expression levels of PI3K and Akt in non-hypertrophic muscles including the soleus of $\mathrm{R}$ rats and the gastrocnemius of $\mathrm{E}$ rats (data not shown), indicated the training-induced selective increases of IGF-1, IGF-1R and mTOR at protein levels in the hypertrophy muscles. Furthermore, as shown in Fig. 7, the selective rises in the activities of PI3K and Akt resulted from the two trainings were decreased by flutamide. In addition, flutamide failed to change the activities of PI3K and Akt of untrained control rats. 


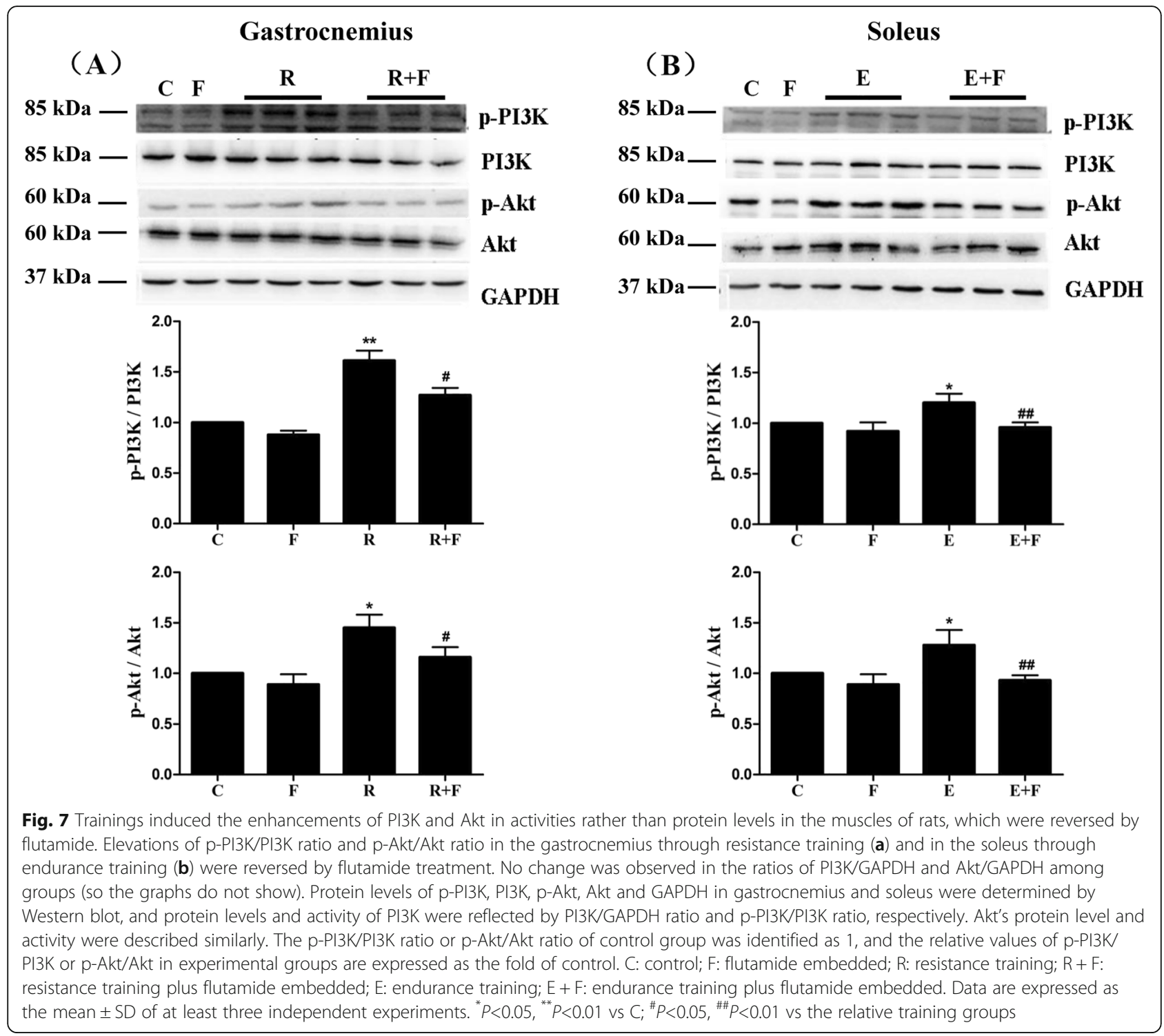

\section{Discussion}

Crucial effects of AR in training-induced selective muscle hypertrophy

The effects of AR on boosting strength and endurance capacity through increasing muscle mass have been further verified in ARKO mice, reflected as a significant decrease of lean mass in multiple muscles including long extensor digitorum, tibialis anterior, gastrocnemius and soleus by $15 \%$ $22 \%$ in global ARKO mice [25] or in hypothalamus ARKO mice [26]. But most of the reports about AR's role were focused on the sedentary participants. At training state, AR was likely to be very important not only for muscle hypertrophy but also strength promotion, for example, the submaximal running endurance and the maximum time to exhaustion were reduced in flutamide-treated rats [10] and in human, the intramuscular AR content influenced the cross-sectional areas of muscle in previously trained men [11]. Although muscle hypertrophy is a vital factor to enhance exercise performance, the roles of AR in exercise-induced muscle hypertrophy are not fully clarified, let alone the underlying molecular mechanisms. In this study, we found that endurance exercise induced the hypertrophy of soleus (typical slow-twitch muscle) while resistance training contributed to the hypertrophy of gastrocnemius (mostly made of type $\Pi$ or fast-twitch fibers), that is training-induced selective muscle hypertrophy. Selective muscle hypertrophy resulted from trainings has been reporting for many years, for example, prolonged resistance training increased the cross-sectional area of type $\Pi$ fiber in frail older adults but not in type I muscle fiber [27], and the abundant type $\Pi$ muscle fibers are usually observed in elite weightlifters [28]. Moreover, in the present study, the training-induced selective muscle hypertrophy and selectively increased AR protein levels were observed, which were 


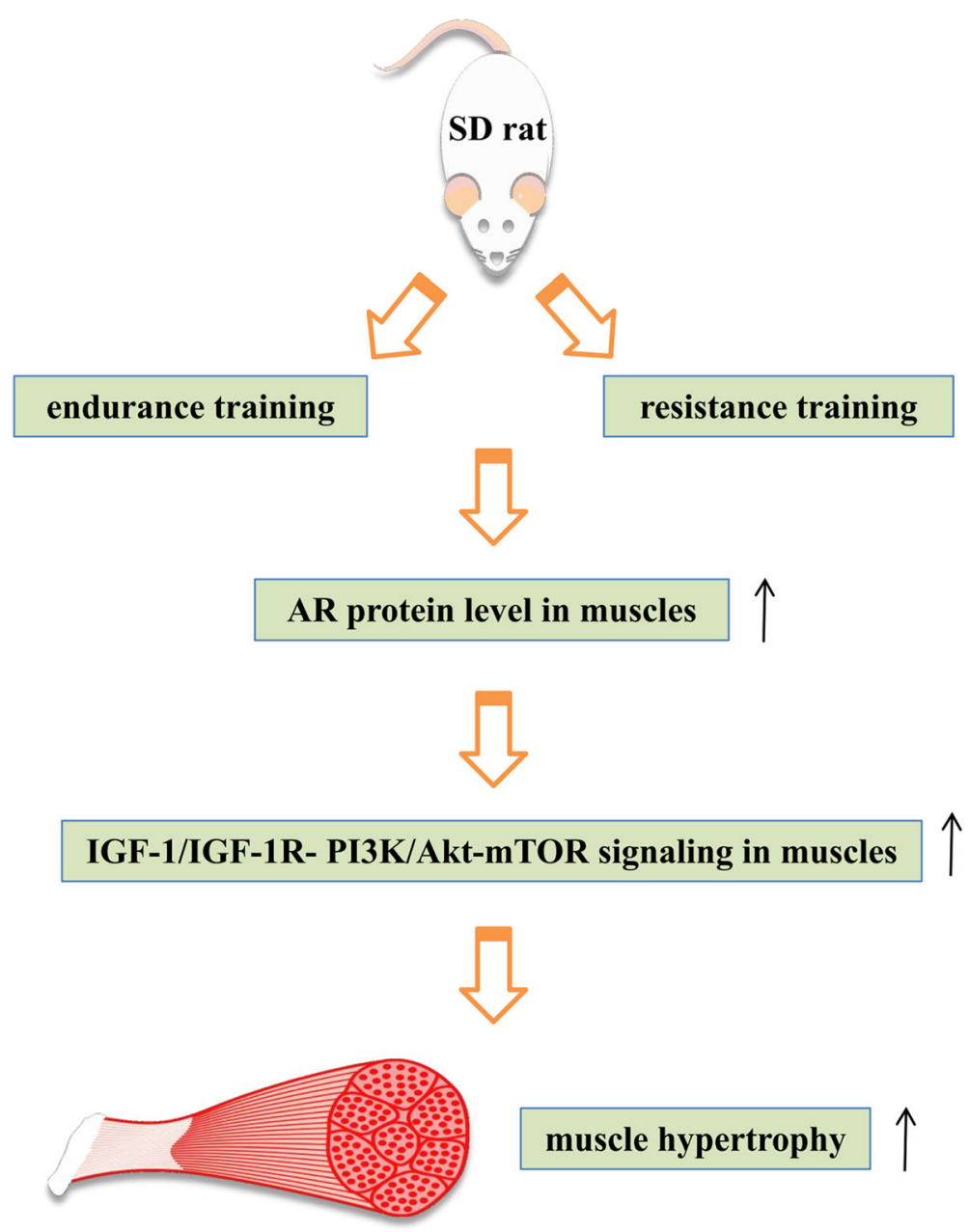

Fig. 8 Summary diagram. Endurance and resistance trainings could raise AR protein levels in the muscle, then activating IGF-1/IGF-1R- PI3K/AktmTOR pathway in the muscle to promote muscle hypertrophy of male rats, which explained the reason in part of training-induced muscle hypertrophy. The thin upward arrowheads stand for increase or promotion

completely reversed by flutamide. Therefore, AR may play an indispensible role in both resistance training and endurance training-related muscle hypertrophy.

In addition, flutamide is considered to inhibit AR singling through blocking AR's binding to its ligand like testosterone or dihydrotestosterone [29], and in the current study flutamide was found to reverse the training-induced increases of AR in muscles, suggested that flutamide might inhibit AR signaling through down-regulating the protein level of AR in tissue, except for the well-known blockade of binding with testosterone. Similar result was reported by a recent study which revealed the inhibitive function of flutamide by downregulating AR protein in tissues [10].

\section{Mechanisms of AR's role in training-induced muscle hypertrophy}

AR affects muscle mass through genomic and nongenomic mechanisms. For genomic mechanism, AR mediates protein synthesis and degradation of skeletal muscle via modulating target genes such as myogenic regulatory factor (MRF) and ubiquitin ligases, which mostly cost more than $24 \mathrm{~h}$ to exert effects for completing transcription and translation of target genes. While the non-genomic mechanism of AR, characteristic of quick response to AR, recently have been proven an increasing importance in muscle protein balance (prompting protein synthesis and inhibiting degradation), is fulfilled through interactions of AR with other molecules such as IGF-1 and myostatin, especially IGF-1 [30].

The important positive effect of IGF-1 on muscle mass was demonstrated in humans including communitydwelling middle-aged and elderly adults [30], and IGF-1 is considered as a promising therapeutic agent for sarcopenia resulted from aging or hypogonadism and muscle weakness from staving [31]. As mentioned in the Introduction, AR's role in increasing muscle mass at unstrained state was mediated by IGF-1/IGF-1R in vivo and in vitro [15], and our 
previous work in two kinds of myoblasts $(\mathrm{C} 2 \mathrm{C} 12$ cells with high AR expression level, while L6 cells without detectable AR) using flutamide, AR over-expression, exogenous IGF-1 and IGF-1R neutralizing antibody (inhibiting IGF-1R signaling) demonstrated that the essential role of AR in myoblasts proliferation resulted from mechanical stretch (mimic muscle movement) was mediated by IGF-1/IGF-1R in vitro $[23,24]$. This study further indicated that traininginduced muscle hypertrophy in vivo was likely to be modulated through IGF-1/IGF-1R.

IGF-1/IGF-1R mainly exert their functions in facilitating proliferation of muscle cells and muscle hypertrophy on untrained or un-stretched state through stimulating PI3K/Akt and thereby activating mTOR [16, 32], and antagonist of PI3K could significantly inhibit the activation of mTOR in $\mathrm{C} 2 \mathrm{C} 12$ cells [33]. For trained state, our previous work in vitro demonstrated the vital effects of IGF-1/IGF-1R on mechanical stretch-induced proproliferation of myoblasts by activating PI3K/AktmTOR using exogenous IGF-1, IGF-1R neutralizing antibody, and inhibitors of PI3K/Akt [23, 24]. In vivo, other researches' evidences suggest the relation between IGF-1/IGF-1R and PI3K/Akt- mTOR because of the synchronous increase during exercise-promoted muscle hypertrophy [34, 35], but needs further verification. In the current study, accompanied with exercise-induced selective muscle hypertrophy, the protein levels of IGF1, IGF-1R and mTOR and the activations of PI3K and Akt were increased only in the hypertrophy muscles but not in the non-hypertrophy muscle (selective enhancements in the levels and activities of the molecules mentioned above), suggested the roles of IGF-1/IGF-1R and PI3K/Akt- mTOR pathway. Furthermore, AR antagonist flutamide could reverse the exercise-induced increases in the protein levels of IGF-1, IGF-1R and mTOR and the activations of PI3K and Akt, accompanied with the reversed muscle hypertrophy. These results indicated that the crucial role of AR in training-induced muscle hypertrophy in vivo might be played through IGF-1/IGF-1R-PI3K/Akt-mTOR pathway. Evidence from other study supports our results, which demonstrated the synergistic effects of androgen/AR signaling on exercise-induced muscle hypertrophy via the activation of mTOR [36]. To our best knowledge, few reports about the relation between AR and PI3K/Akt- mTOR pathway in trained state in vivo has been found.

To sum up (Fig. 8), the present study firstly demonstrated a crucial effect of AR on either resistance or endurance training-induced muscle hypertrophy in vivo, and further suggested that AR's role might be achieved through the mediation of IGF-1/IGF-1R-PI3K/Akt-mTOR pathway, which facilitates the understandings on the roles and mechanisms of $\mathrm{AR}$ in exercise-induced increases of muscle hypertrophy. However, a limitation that we have to consider in this study is that we have not demonstrated the indispensability of PI3K/
Akt- mTOR pathway for AR's roles in exercise-induced muscle hypertrophy, and inhibitors of PI3K, Akt and mTOR need to be used in rats in the future to clarify that question.

\section{Conclusions}

AR played a crucial role in both resistance and endurance trainings-induced muscle hypertrophy of rats, which might be achieved at least partly through the mediation of IGF-1/IGF-1R- PI3K/Akt- mTOR pathway.

\section{Abbreviations}

AR: Androgen receptor; IGF-1: Insulin-like growth factor-1; mTOR: Mammalian target of rapamycin; PI3K: Phosphatidylinositol 3 kinase; Akt: Protein kinase B; ARKO: Androgen receptor gene knockout; MRF: Myogenic regulatory factor

\section{Acknowledgements}

We gratefully acknowledge the grants for National Natural Science Foundation of China (No.31872801) and Shanghai Key Lab of Human Performance (Shanghai University of Sport) (No. 11DZ2261100).

\section{Authors' contributions}

Lijun Yin, Lin Lu and Xiaojing Lin performed experiments, analyzed data and interpreted results of experiments; Lijun Yin drafted manuscript, and Xiaohui Wang designed, edited and revised the manuscript. All authors have read and approved the final version of the manuscript.

\section{Funding}

This work is supported by grants from the National Natural Science Foundation of China (No.31872801) and from Shanghai Key Lab of Human Performance (Shanghai University of Sport) (No. 11DZ2261100).

\section{Availability of data and materials}

The datasets analysed in this study are available from the corresponding author upon reasonable request.

Ethics approval and consent to participate

The animal protocol was approved and all the experimental procedures were supervised by the Ethics Committee of Shanghai University of Sport (No. 2018002).

\section{Consent for publication}

Not applicable.

\section{Competing interests}

We declare that we have no competing interests.

Received: 25 December 2019 Accepted: 21 March 2020

Published online: 30 March 2020

\section{References}

1. Shukla GC, Plaga AR, Shankar E, et al. Androgen receptor-related diseases: what do we know? [J]. Andrology. 2016;4(3):366-81. https://doi.org/10.1111/ andr.12167.

2. Park HJ, Ahn ST, Moon DG. Evolution of guidelines for testosterone replacement therapy [J]. J Clin Med. 2019;8(3):410. https://doi.org/10.3390/ jcm8030410.

3. Solomon ZJ, Mirabal JR, Mazur DJ, et al. Selective androgen receptor modulators: current knowledge and clinical applications [J]. Sex Med Rev. 2019;7(1):84-94. https://doi.org/10.1016/j.sxmr.2018.09.006.

4. Ponnusamy S, Sullivan $\mathrm{RD}$, You D, et al. Androgen receptor agonists increase lean mass, improve cardiopulmonary functions and extend survival in preclinical models of Duchenne muscular dystrophy [J]. Hum Mol Genet. 2017;26(13):2526-40. https://doi.org/10.1093/hmg/ddx150.

5. Clarke MV, Russell PK, Zajac JD, et al. The androgen receptor in the hypothalamus positively regulates hind-limb muscle mass and voluntary physical activity in adult male mice [J]. J Steroid Biochem Mol Biol. 2019;189: 187-94. https://doi.org/10.1016/j.jsbmb.2019.02.018.

6. Wu Y, Yang HF, Wang $X \mathrm{H}$. The function of androgen/androgen receptor and insulin growth factor 1 /insulin growth factor 1 receptor on the effects 
of Tribulus terrestris extracts in rats undergoing high intensity exercise [J]. Mol Med Rep. 2017;16(3):2931-8. https://doi.org/10.3892/mmr.2017.6891.

7. Dubois V, Laurent MR, Jardi F, et al. Androgen deficiency exacerbates high fat diet-induced metabolic alterations in male mice [J]. Endocrinology. 2016; 157(2):648-65. https://doi.org/10.1210/en.2015-1713.

8. Dubois V, Laurent MR, Sinnesael M, et al. A satellite cell-specific knockout of the androgen receptor reveals myostatin as a direct androgen target in skeletal muscle [J]. FASEB J. 2014;28(7):2979-94.

9. Roberts MD, Haun $C T$, Mobley $C B$, et al. Physiological differences between low versus high skeletal muscle hypertrophic responders to resistance exercise training: current perspectives and future research directions [J]. Front Physiol. 2018;9:834. https://doi.org/10.3389/fphys.2018.00834

10. Georgieva KN, Angelova PA, Gerginska FD, et al. The effect of flutamide on the physical working capacity and activity of some of the key enzymes for the energy supply in adult rats []]. Asian J Androl. 2017;19(4):444-8 http:// www.ajandrology.com/text.asp?2017/19/4/444/177842.

11. Morton RW, Sato K, Gallaugher MPB, et al. Muscle androgen receptor content but not systemic hormones is associated with resistance traininginduced skeletal muscle hypertrophy in healthy, young men [J]. Front Physiol. 2018;9:1373. https://doi.org/10.3389/fphys.2018.01373.

12. Pronsato L, Milanesi L, Vasconsuelo A. Testosterone induces up-regulation of mitochondrial gene expression in murine C2C12 skeletal muscle cells accompanied by an increase of nuclear respiratory factor- 1 and its downstream effectors [J]. Mol Cell Endocrinol. 2020;500:110631. https://doi. org/10.1016/j.mce.2019.110631.

13. Kupreeva M, Diane A, Lehner R, et al. Effect of metformin and flutamide on insulin, lipogenic and androgen-estrogen signaling, and cardiometabolic risk in a PCOS-prone metabolic syndrome rodent model [J]. Am J Physiol Endocrinol Metab. 2019;316(1):E16-33. https://doi.org/10.1152/ajpendo.00018.2018.

14. JG MK, Yaden BC, Bullock $\mathrm{H}$, et al. Molecular targets of androgen signaling that characterize skeletal muscle recovery and regeneration.[J]. Nucl Recept Signal. 2015;13(1):13005. https://doi.org/10.1621/nrs.13005.

15. Hughes DC, Stewart CE, Sculthorpe N, et al. Testosterone enables growth and hypertrophy in fusion impaired myoblasts that display myotube atrophy: deciphering the role of androgen and IGF-I receptors [J]. Biogerontology. 2016; 17(3):619-39. https://doi.org/10.1007/s10522-015-9621-9.

16. Rhoads RP, Baumgard LH, El-Kadi SW, et al. Phisiology and endocrinology symposium: roles for insulin-supported skeletal muscle growth [J]. J Anim Sci. 2016;5(94):1791-802. https://doi.org/10.2527/jas.2015-0110.

17. $\mathrm{Ma} \mathrm{BB}, \mathrm{He} X \mathrm{XF}, \mathrm{Lu} Z$ Z, et al. Chronic heat stress affects muscle hypertrophy, muscle protein synthesis and uptake of amino acid in broilers via insulin like growth factor-mammalian target of rapamycin signal pathway []]. Poult Sci. 2018;97(12):4150-8. https://doi.org/10.3382/ps/pey291.

18. Timmer LT, Hoogaars WMH, Jaspers RT. The role of IGF-1 signaling in skeletal muscle atrophy [J]. Adv Exp Med Biol. 2018;1088:109-37. https://doi. org/10.1007/978-981-13-1435-3_6.

19. Yu M, Wang $\mathrm{H}$, Xu Y, et al. Insulin-like growth factor-1 (IGF-1) promotes myoblast proliferation and skeletal muscle growth of embryonic chickens via the PI3K Akt signalling pathway [J]. Cell Biol Int. 2015;39(8):910-22. https://doi.org/10.1002/cbin.10466.

20. Dubois V, Laurent M, Boonen S, et al. Androgens and skeletal muscle cellular and molecular action mechanisms underlying the anabolic actions []]. Cell Mol Life Sci. 2012;69(10):1651-67. https://doi.org/10.1007/s00018-011-0883-3.

21. Basualto-Alarcón C, Jorquera G, Altamirano F, et al. Testosterone signals through mTOR and androgen receptor to induce muscle hypertrophy [J]. Med Sci Sports Exerc. 2013;45(9):1712-20. https://doi.org/10.1249/MSS. 0b013e31828cf5f3.

22. Eane CS, Hughes DC, Sculthorpe N, et al. Impaired hypertrophy in myoblasts is improved with testosterone administration [J]. J Steroid Biochem Mol Biol. 2013;138:152-61. https://doi.org/10.1016/j.jsbmb.2013.05.005.

23. Ma Y, Fu S, Lu L, et al. Role of androgen receptor on cyclic mechanical stretch-regulated proliferation of $\mathrm{C} 2 \mathrm{C} 12$ myoblasts and its upstream signals: IGF-1-mediated PI3K/Akt and MAPKs pathways [J]. Mol Cell Endocrinol. 2017; 450:83-93. https://doi.org/10.1016/.mce.2017.04.021.

24. Fu S, Yin L, Lin X, et al. Effects of cyclic mechanical stretch on the proliferation of $\mathrm{L} 6$ myoblasts and its mechanisms: PI3K/Akt and MAPK signal pathways regulated by IGF-1 receptor [J]. Int J Mol Sci. 2018;19(6):1649. https://doi.org/10.3390/ijms19061649.

25. Rana K, Davey RA, Zajac JD. Human androgen deficiency: insights gained from androgen receptor knockout mouse models [J]. Asian J Androl. 2014; 16(2):169-77. https://doi.org/10.4103/1008-682X.122590.
26. Davey RA, Clarke MV, Russell PK, et al. Androgen action via the androgen receptor in neurons within the brain positively regulates muscle mass in male mice []]. Endocrinology. 2017;158(10):3684-95. https//doi.org/10.1210/en.2017-00470.

27. Dirks ML, Tieland M, Verdijk LB, et al. Protein supplementation augments muscle fiber hypertrophy but does not modulate satellite cell content during prolonged resistance-type exercise training in frail elderly [J]. J Am Med Dir Assoc. 2017;18(7):608-15. https://doi.org/10.1016/j.jamda.2017.02.006.

28. Serrano N, Colenso-Semple LM, Lazauskus KK, et al. Extraordinary fast-twitch fiber abundance in elite weightlifters [J]. PLoS One. 2019;14(3):e0207975. https://doi.org/10.1371/journal.pone.0207975.

29. Hejmej A, Bilinska B. The effects of flutamide on cell-cell junctions in the testis, epididymis, and prostate [J]. Reprod Toxicol. 2018:81:1-16. https://doi. org/10.1016/j.reprotox.2018.06.014.

30. Moriwaki K, Matsumoto H, Tanishima S, et al. Association of serum boneand muscle-derived factors with age, sex, body composition, and physical function in community-dwelling middle-aged and elderly adults: a crosssectional study [J]. BMC Musculoskelet Disord. 2019;20(1):276. https://doi. org/10.1186/s12891-019-2650-9

31. Ascenzi F, Barberi L, Dobrowolny G, et al. Effects of IGF-1 isoforms on muscle growth and sarcopenia [J]. Aging Cell. 2019;18(3):e12954. https://doi. org/10.1111/acel.12954.

32. Pratiwi YS, Lesmana $R$, Goenawan $H$, et al. Nutmeg extract increases skeletal muscle mass in aging rats partly via IGF1-AKT-mTOR pathway and inhibition of autophagy [J]. Evid Based Complement Alternat Med : eCAM. 2018;2018: 1-8. https://doi.org/10.1155/2018/2810840.

33. Kitakaze T, Sakamoto T, Kitano T, et al. The collagen derived dipeptide hydroxyprolyl-glycine promotes $\mathrm{C} 2 \mathrm{C} 12$ myoblast differentiation and myotube hypertrophy [J]. Biochem Biophys Res Commun. 2016;478(3):12927. https://doi.org/10.1016/j.bbrc.2016.08.114

34. Palstra AP, Rovira M, Rizo-Roca D, et al. Swimming-induced exercise promotes hypertrophy and vascularization of fast skeletal muscle fibres and activation of myogenic and angiogenic transcriptional programs in adult zebrafish [J]. BMC Genomics. 2014;15(1):1136. https://doi.org/10.1186/14712164-15-1136

35. Launay T, Momken I, Carreira S, et al. Acceleration-based training: a new mode of training in senescent rats improving performance and left ventricular and muscle functions [J]. Exp Gerontol. 2017;95:71-6. https://doi. org/10.1016/j.exger.2017.05.002

36. Zeng $\mathrm{F}$, Zhao $\mathrm{H}$, Liao J. Androgen interacts with exercise through the mTOR pathway to induce skeletal muscle hypertrophy [J]. Biol Sport. 2017;34(4): 313-21. https://doi.org/10.5114/biolsport.2017.69818.

\section{Publisher's Note}

Springer Nature remains neutral with regard to jurisdictional claims in published maps and institutional affiliations.

Ready to submit your research? Choose BMC and benefit from:

- fast, convenient online submission

- thorough peer review by experienced researchers in your field

- rapid publication on acceptance

- support for research data, including large and complex data types

- gold Open Access which fosters wider collaboration and increased citations

- maximum visibility for your research: over $100 \mathrm{M}$ website views per year

At $\mathrm{BMC}$, research is always in progress.

Learn more biomedcentral.com/submissions 